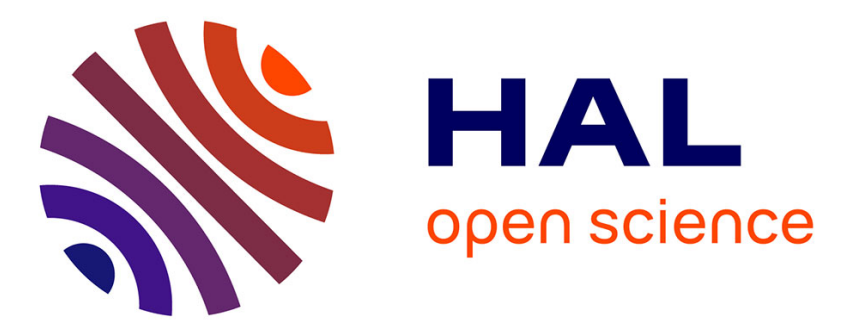

\title{
Semianalytical evaluation of linear and nonlinear piezoelectric potentials for quantum nanostructures with axial symmetry
}

Jacky Even, François Doré, Charles Cornet, Laurent Pedesseau, Andrei Schliwa, Dieter Bimberg

\section{To cite this version:}

Jacky Even, François Doré, Charles Cornet, Laurent Pedesseau, Andrei Schliwa, et al.. Semianalytical evaluation of linear and nonlinear piezoelectric potentials for quantum nanostructures with axial symmetry. Applied Physics Letters, 2007, pp.122112. 10.1063/1.2787894 . hal-00492292

\section{HAL Id: hal-00492292 \\ https://hal.science/hal-00492292}

Submitted on 15 Jun 2010

HAL is a multi-disciplinary open access archive for the deposit and dissemination of scientific research documents, whether they are published or not. The documents may come from teaching and research institutions in France or abroad, or from public or private research centers.
L'archive ouverte pluridisciplinaire HAL, est destinée au dépôt et à la diffusion de documents scientifiques de niveau recherche, publiés ou non, émanant des établissements d'enseignement et de recherche français ou étrangers, des laboratoires publics ou privés. 


\title{
Semi-analytical evaluation of linear and non-linear piezoelectric potential for quantum nanostructures with axial symmetry
}

\author{
J. Even*, F. Doré*, C. Cornet*, L. Pedesseau*, A. Schliwa ${ }^{\text {T }}$ and D. Bimberg $^{\text {T }}$ \\ *FOTON-INSA Laboratory, UMR 6082 au CNRS, INSA de Rennes, 20 Avenue des Buttes de \\ Coësmes, CS 14315, 35043 Rennes Cedex, France \\ ${ }^{\mathrm{T}}$ Institut für Festkörperphysik, Technische Universität Berlin, Hardenbergstrasse 36, 10623 \\ Berlin, Germany
}

corresponding author : jacky.even@insa-rennes.fr (J. Even)

\begin{abstract}
.
A model is proposed to describe the linear and quadratic piezoelectric potentials in quantum nanostructures with axial symmetry. Semi-analytical expressions of the charge densities are given to illustrate the symmetry reduction. The results are compared for InAs/InP QD to previous full $3 \mathrm{D}$ calculation and to the full isotropic elastic model. The model is well-adapted to study various effects for quantum dots (QD): the lattice mismatch, the QD materials composition or alloying, the QD size or shape. Special emphasis is put on variations of the vertical aspect ratio and the role of the wetting layer.
\end{abstract}

P.A.C.S. 71.20.Nr, 73.21.La, 78.20.Hp 
The anisotropic strain in and around of zinc-blende based semiconductor quantum dots (QD) can generate local piezoelectric charges ${ }^{1}$. If the QDs are grown on (001) substrate and their axial symmetry is larger than $\mathrm{C}_{2 \mathrm{v}}$ the resulting piezoelectric potential gives rise to a symmetry reduction of the electronic confinement potential. As a result, many degeneracies of the single particle energies, like the electron p-states, are lifted and the excitonic bright states are split ${ }^{2}$. The proper handling of the piezoelectric fields is an essential prerequisite for the electronic structure simulation, where the $\mathrm{kp}$ method $^{1,3,4}$ matured to a standard method although more elaborate theoretical schemes can be employed ${ }^{5-7}$.

Recently it has been shown ${ }^{6,7}$ that quadratic piezoelectric effect should be taken into account and may be greater than the linear one. QD geometries with axial symmetry give a good opportunity for testing the expected symmetry reduction from $C_{\infty \mathrm{v}}$ to $\mathrm{C}_{2 \mathrm{v}}{ }^{6,7}$. This geometry is also useful to describe practical cases ${ }^{4}$. We propose in this work to take into account linear and quadratic piezoelectric effects in quantum nanostructures with a semianalytical approach based on continuum elasticity and an axial approximation for elastic constants. We will show that most symmetry features are captured by this model. Examples of strain and piezoelectric potential calculation will be given for $\operatorname{InAs} / \mathrm{InP}$, InAs/GaAs and InAs/GaP QD. The lattice mismatches are respectively equal to $3.2 \%, 7.2 \%$ and $11.2 \%$.

In this paper we examine QD geometries corresponding to the $\mathrm{C}_{\infty \mathrm{v}}$ symmetry (rotational symmetry around the z-[001]-axis). As an example figure 1-a shows a 2D view of a truncated cone QD with a wetting layer (WL). The real calculations throughout this work are performed in cylindrical coordinates $(\boldsymbol{r}, \mathbf{z})$. Cones or truncated cones are considered in the following, with or without a WL. The reference for the $\mathrm{z}$ axis and for the QD height is the bottom of the WL. The chosen dimensions are $8.8 \mathrm{~nm}$ for the cone height, $1.2 \mathrm{~nm}$ for the WL 
thickness and $17.5 \mathrm{~nm}$ for the radius. The A) B) C) horizontal dotted lines correspond to the cross sections of the piezoelectric potential displayed in figure 3 for $z=10.5 \mathrm{~nm}, 5.4 \mathrm{~nm}$ and $2.5 \mathrm{~nm}$, respectively.

If the strain field is calculated using a continuum mechanical model (elasticity) for the zinc-blende crystal, the geometry of the deformed QD has a $\mathrm{C}_{4 \mathrm{v}}$ symmetry slightly deviating from the $\mathrm{C}_{\infty \mathrm{v}}$ symmetry on the (100) substrate. Our approach is based on an "axial approximation" for the strain field. This approximation consists in replacing the elastic moduli $C_{44}$ and $\frac{C_{11}-C_{12}}{2}$ by an effective modulus $\bar{C}$ in the expression of the stress tensor obtained by an arbitrary rotation around the $\mathrm{z}$ axis. The elastic moduli are then the same for any frame chosen around the $\mathrm{z}$ axis:

$$
\begin{aligned}
& \left(\begin{array}{cccccc}
\boldsymbol{C}_{11}^{\prime} & \boldsymbol{C}_{12}^{\prime} & \boldsymbol{C}_{12} & 0 & 0 & 0 \\
\boldsymbol{C}_{12}^{\prime} & \boldsymbol{C}_{11}^{\prime} & \boldsymbol{C}_{12} & 0 & 0 & 0 \\
\boldsymbol{C}_{12} & \boldsymbol{C}_{12} & \boldsymbol{C}_{11} & 0 & 0 & 0 \\
0 & 0 & 0 & \boldsymbol{C}_{44} & 0 & 0 \\
0 & 0 & 0 & 0 & \boldsymbol{C}_{44} & 0 \\
0 & 0 & 0 & 0 & 0 & \overline{\boldsymbol{C}}
\end{array}\right) \text { with } \boldsymbol{C}_{11}^{\prime}=\frac{\boldsymbol{C}_{11}+\boldsymbol{C}_{12}}{2}+\overline{\boldsymbol{C}}, \boldsymbol{C}_{12}^{\prime}=\frac{\boldsymbol{C}_{11}+\boldsymbol{C}_{12}}{2}-\overline{\boldsymbol{C}} \\
& \text { and } \overline{\boldsymbol{C}}=\frac{\boldsymbol{C}_{11}-\boldsymbol{C}_{12}}{2}+\left(\boldsymbol{C}_{44}-\frac{\boldsymbol{C}_{11}-\boldsymbol{C}_{12}}{2}\right) \boldsymbol{d}
\end{aligned}
$$

This is a special case, with four independent parameters $\left(\boldsymbol{C}_{11}, \boldsymbol{C}_{12}, \boldsymbol{C}_{44}, \overline{\boldsymbol{C}}\right)$, of the "transverse isotropic" material type with corresponds to five independent parameters in the general case. For $\boldsymbol{d}=0$ or $\boldsymbol{d}=1$, there are even only three independent parameters $\left(\boldsymbol{C}_{11}, \boldsymbol{C}_{12}, \boldsymbol{C}_{44}\right)$. We may notice that it is not necessary to replace everywhere $\boldsymbol{C}_{44}$ by $\frac{C_{11}-C_{12}}{2}$ like in the full isotropic approximation ${ }^{8}$. In principle, $\bar{C}$ can take any positive value, but a good choice is $\bar{C}=\frac{C_{11}-C_{12}}{2}(\boldsymbol{d}=0)$ as shown in figure 2 . In that case, only one 
elastic constant $\left(\boldsymbol{C}_{66}\right)$ is changed by comparison to the zinc-blende crystal. The biaxial deformation for a QW (like the WL) remains equal to $\frac{-2 C_{12}}{C_{11}}$ times the lattice mismatch.

It is convenient to use the components of the strain tensor $\varepsilon_{r r}, \varepsilon_{\varphi \varphi}, \varepsilon_{z z}, \varepsilon_{r z}$ in the cylindrical representation $(\boldsymbol{r}, \boldsymbol{\varphi}, \mathbf{z})$ :

$$
\begin{gathered}
\varepsilon_{x x}=\cos ^{2}(\varphi) \varepsilon_{r r}+\sin ^{2}(\varphi) \varepsilon_{\varphi \varphi}, \varepsilon_{y y}=\sin ^{2}(\varphi) \varepsilon_{r r}+\cos ^{2}(\varphi) \varepsilon_{\varphi \varphi} \\
\varepsilon_{x z}=\cos (\varphi) \varepsilon_{r z}, \varepsilon_{y z}=\sin (\varphi) \varepsilon_{r z}, \varepsilon_{x y}=\frac{\sin (2 \varphi)}{2}\left(\varepsilon_{r r}-\varepsilon_{\varphi \varphi}\right)
\end{gathered}
$$

When considering now the linear piezoelectric polarization $\overrightarrow{\boldsymbol{P}}_{1}$ and charge density $\boldsymbol{\rho}_{1}$, it is straightforward to show that:

$$
\begin{gathered}
\overrightarrow{\mathrm{P}}_{1}=2 \mathrm{e}_{14}\left[\sin (2 \varphi) \varepsilon_{\mathrm{rz}} \overrightarrow{\mathrm{u}}_{\mathrm{r}}+\cos (2 \varphi) \varepsilon_{\mathrm{rz}} \overrightarrow{\mathrm{u}}_{\varphi}+\frac{\sin (2 \varphi)}{2}\left(\varepsilon_{\mathrm{rr}}-\varepsilon_{\varphi \varphi}\right) \overrightarrow{\mathrm{u}}_{\mathrm{z}}\right] \\
\rho_{1}=-\frac{\mathrm{e}_{14} \sin (2 \varphi)}{\varepsilon_{0}}\left[\frac{1}{\mathrm{r}} \frac{\partial\left[2 \mathrm{r} \varepsilon_{\mathrm{rz}}\right]}{\partial \mathrm{r}}+\frac{4 \varepsilon_{\mathrm{rz}}}{\mathrm{r}}+\frac{\partial\left[\left(\varepsilon_{\mathrm{rr}}-\varepsilon_{\varphi \varphi}\right)\right]}{\partial \mathrm{z}}\right]
\end{gathered}
$$

Associated Coulomb potential has automatically a semi-analytical expression where the simple angular dependence corresponds to the introduction of $\mathrm{C}_{2 \mathrm{v}}$ symmetry $V_{1}(r, \varphi, z)=\sin (2 \varphi) f_{1}(r, z)$. Determination of $\boldsymbol{f}_{1}(\boldsymbol{r}, \mathbf{z})$ is performed using a fast finite element resolution of the 2D Poisson's equation with a commercial software ${ }^{9}$. Figures 1-b and 1-c compare the linear piezoelectric field for the same InAs/GaAs conic QD geometry without a WL (figure 1-b) or with a WL (figure 1-c). It should be pointed out that the chosen linear piezoelectric potential for these calculations are the experimental ones (InAs $\boldsymbol{e}_{14}=-0.045 \mathrm{Cm}^{-2}$, GaAs $\left.\boldsymbol{e}_{14}=-0.16 \mathrm{Cm}^{-2}\right)$. The linear piezoelectric potential reaches higher maximum values without a WL than with a WL. It is also larger for QDs on the GaAs substrate than on the InP substrate. This is related to the increase of the lattice mismatch 
between the InP and the GaAs substrates. The magnitude of the linear piezoelectric coefficient $\boldsymbol{e}_{14}$ is also important for this effect, which is particularly true for GaAs. The maximum of the potential is on the same order for InAs/GaP QD than for InAs/GaAs QD.

Figure 2 compares the results obtained using various theoretical approaches for the same InAs/InP QD with a WL. The maximum piezoelectric potential values above the QD plane is calculated as a function of the truncation height $h$, with a full 3D model (our previous work ${ }^{4}$ ), a full isotropic model and our transverse isotropic model with $\boldsymbol{d}=0$ and $\boldsymbol{d}=1$. The best agreement with the full 3D model is obtained for the transverse isotropic model with $\boldsymbol{d}=0$. We have added the results of a systematic calculation without a WL. In that case the potential is larger than for a QD with a WL, like in the InAs/GaAs case (figure 1).

Figure 3 is a comparison between the results obtained in this work for a conic InAs/InP without a WL (left hand side) and the ones obtained for the same geometry with a WL in our previous work $^{4}$ (full 3D calculation, right hand side). The results obtained in this work for the same geometry with a WL are similar to the ones presented on the right hand side and are not represented. This figure shows cross sections of the piezoelectric potential above the QD (a), inside the QD (b) and below the QD (c). It appears that the complex variations of the piezoelectric potential are very well reproduced with the $2 \mathrm{D}$ semi-analytical model. The $\sin (2 \varphi)$ angular variation of the $2 \mathrm{D}$ solution captures most of the features coming from the $\mathrm{C}_{2 \mathrm{v}}$ symmetry. The amplitude of the piezoelectric potential on the left hand side is larger than on the right hand side due to the WL effect.

Quadratic piezoelectric polarization $\overrightarrow{\boldsymbol{P}}_{2}$ and charge density $\boldsymbol{\rho}_{2}{ }^{6,7}$, are evaluated using the same procedure :

$$
\overrightarrow{\mathrm{P}}_{2}=\mid \begin{gathered}
\sin (2 \varphi)\left[\mathrm{B}_{114}\left(\varepsilon_{\mathrm{rr}}+\varepsilon_{\varphi \varphi}\right) \varepsilon_{\mathrm{rz}}+\mathrm{B}_{124}\left(\varepsilon_{\mathrm{rr}}+\varepsilon_{\varphi \varphi}+2 \varepsilon_{\mathrm{zz}}\right) \varepsilon_{\mathrm{rz}}+2 \mathrm{~B}_{156}\left(\varepsilon_{\mathrm{rr}}-\varepsilon_{\varphi \varphi}\right) \varepsilon_{\mathrm{rz}}\right] \overrightarrow{\mathrm{u}}_{\mathrm{r}} \\
2 \cos (2 \varphi)\left[\mathrm{B}_{114} \varepsilon_{\varphi \varphi} \varepsilon_{\mathrm{rz}}+\mathrm{B}_{124}\left(\varepsilon_{\mathrm{rr}}+\varepsilon_{\mathrm{zz}}\right) \varepsilon_{\mathrm{rz}}\right] \overrightarrow{\mathrm{u}}_{\varphi} \\
\sin (2 \varphi)\left[\mathrm{B}_{114}\left(\varepsilon_{\mathrm{rr}}-\varepsilon_{\varphi \varphi}\right) \varepsilon_{\mathrm{zz}}+\mathrm{B}_{124}\left(\varepsilon_{\mathrm{rr}}^{2}-\varepsilon_{\varphi \varphi}{ }^{2}\right)+2 \mathrm{~B}_{156} \varepsilon_{\mathrm{rz}}{ }^{2}\right] \overrightarrow{\mathrm{u}}_{\mathrm{z}}
\end{gathered}
$$




$$
\rho_{2}=\frac{-\operatorname{div}\left(\overrightarrow{\boldsymbol{P}}_{2}\right)}{\varepsilon_{0}}
$$

The total piezoelectric potential $\boldsymbol{V}(\boldsymbol{r}, \boldsymbol{\varphi}, \boldsymbol{z})=\sin (2 \boldsymbol{\varphi}) \boldsymbol{f}(\boldsymbol{r}, \boldsymbol{z})$ is calculated by adding linear and quadratic charge densities. We may note that the angular modulation of the potential $\left(\mathrm{C}_{2 \mathrm{v}}\right.$ symmetry) is unchanged by the quadratic effect and that only the amplitude $f(r, z)$ is subject to change.

Our semi-analytical model is convenient to study various effects (materials, size of the QD, lattice mismatch ...) and also to compare the piezoelectric potentials computed using either experimental or $a b$ initio values. We should point out that the linear piezoelectric coefficients presented in ref. 6, 7 are larger than the classical ones we are using ( $\operatorname{InAs} \mathrm{e}_{14}=-0.115 \mathrm{Cm}^{-2}$, $\left.\mathrm{GaP} \mathrm{e}_{14}=-0.15 \mathrm{Cm}^{-2}\right)$. We have used the same procedure to evaluate the quadratic piezoelectric coefficients for $\mathrm{GaP}$ using $a b$ initio calculations (abinit software) ${ }^{10}$ : $\left(\operatorname{InAs} \mathrm{s}^{6,7}\right.$ $\mathrm{B}_{114}=-0.531 \mathrm{Cm}^{-2} \mathrm{~B}_{124}=-4.076 \mathrm{Cm}^{-2} \mathrm{~B}_{156}=-0.120 \mathrm{Cm}^{-2} \quad, \quad \mathrm{GaP} \quad \mathrm{B}_{114}=-0.762 \mathrm{Cm}^{-2}$ $\left.\mathrm{B}_{124}=-3.562 \mathrm{Cm}^{-2} \mathrm{~B}_{156}=-0.975 \mathrm{Cm}^{-2}\right)$. Figure 4 is an illustration of this comparison for InAs/GaP QD. Isosurfaces for $\pm 50 \mathrm{mV}$ values of piezoelectric potential are represented in the linear case $\left(\mathrm{P}_{1}\right)$ a), the quadratic piezoelectric potential case $\left(\mathrm{P}_{2}\right)$ b), the sum of both contributions $\left.\left(\mathrm{P}_{1}+\mathrm{P}_{2}\right) \mathrm{c}\right)$ and the linear piezoelectric potential calculated with experimental constants $\left.\left(\mathrm{P}_{\mathrm{ex}}\right) \mathrm{d}\right)$. The structure of the piezoelectric potential becomes more complex when the quadratic term is added. It is clear that the total piezoelectric potential is now affected by rapid variation of the QD shape close to the edges. This variation is due to the quadratic term which is much more sensitive to strain concentration. Former computational studies ${ }^{1,5,7}$ have shown that the electronic fine structure of QD is modified by the piezoelectric field. We 
conclude that the fluctuations of the electronic fine structure may be associated in real systems to the fluctuation of strains and quadratic piezoelectric effect near edges. Indeed, it is well-known that an inhomogeneous dispersion of QD size, shape or alloying is obtained for self-assembled QD. This semi-analytical model can be used for any quantum nanostructure within the axial approximation. 


\section{References}

${ }^{1}$ O. Stier, M. Grundmann and D. Bimberg, Phys. Rev. B59, 5688 (1999)

${ }^{2}$ R. Seguin, A. Schliwa, S.Rodt, K.Pötschke, U.W.Pohl, and D.Bimberg, Phys. Rev. Lett. 95, 257402, (2005).

${ }^{3}$ C. Pryor, Phys. Rev. B57, 7190 (1998)

${ }^{4}$ C. Cornet, A. Schliwa, J. Even, F. Dore, C. Celebi, A. Letoublon, E. Mace, C. Paranthoen, A. Simon, P. M. Koenraad, N. Bertru, D. Bimberg and S. Loualiche, Phys. Rev. B74 035312 (2006)

${ }^{5}$ G. Bester and A. Zunger, Phys. Rev. B71 045318 (2005)

${ }^{6}$ G. Bester, X. Wu, D. Vanderbilt and A. Zunger,, Phys. Rev. Lett. 96187602 (2006)

${ }^{7}$ G. Bester, A. Zunger, X. Wu and D. Vanderbilt, Phys. Rev. B74 081305(R) (2006)

${ }^{8}$ M. Tadic, F.M. Peeters and K. J. Janssens, Phys. Rev. B65, 165333 (2002)

${ }^{9}$ Femlab 2.0 software, Trademark of Comsol AB (2000).

${ }^{10}$ X. Gonze, J.-M. Beuken, R. Caracas, F. Detraux, M. Fuchs, G.-M. Rignanese, L. Sindic, M. Verstraete, G. Zerah, F. Jollet, M. Torrent, A. Roy, M. Mikami, Ph. Ghosez, J.-Y. Raty, D.C. Allan. Computational Materials Science 25, 478-492 (2002). 


\section{Figure captions}

Figure $1:$ a) Schematic view of a truncated cone QD with a WL. The reference for the $\mathrm{z}$ axis and for the QD height is the bottom of the WL (indicated by an arrow). The chosen dimensions are $8.8 \mathrm{~nm}$ for the cone height, $1.2 \mathrm{~nm}$ for the WL thickness and $17.5 \mathrm{~nm}$ for the radius. The A) B) C) horizontal dotted lines correspond to the cross sections of the piezoelectric potential as displayed in figure 3 for $\mathrm{z}=10.5 \mathrm{~nm}, 5.4 \mathrm{~nm}$ and $-2.5 \mathrm{~nm}$ respectively

b) Linear piezoelectric potential for a conical InAs/GaAs QD without a WL

c) Linear piezoelectric potential for a conical InAs/GaAs QD with a WL

Figure 2 : Comparison between the results obtained using various theoretical approaches for the same InAs/InP QD with a WL. The maximum piezoelectric potential values above the QD plane is calculated as a function of the truncation height $h$ : with a full $3 \mathrm{D}$ model (our previous work $^{4}$ ), a full isotropic model and a transverse isotropic model with $\boldsymbol{d}=0$ and $\boldsymbol{d}=1$. The results of a systematic calculation without a WL are added for $\boldsymbol{d}=0$.

Figure 3 : Plane projections of the piezoelectric potential for an InAs QD with a conical shape embedded in an InP matrix, taking into account only the linear effect. The a) b) c) cross sections corresponds to $\mathrm{z}=10.5 \mathrm{~nm}, 5.4 \mathrm{~nm}$ and $-2.5 \mathrm{~nm}$ respectively (see figure 1 ). The pictures on the left hand side are obtained in this work for a QD without a WL, whereas the pictures on the right hand side were obtained for a QD with a WL by a full 3D calculation in our previous work (ref. 4). 
Figure 4 : Isosurfaces for $\pm 50 \mathrm{mV}$ values of the piezoelectric potential in a conic $\mathrm{InAs} / \mathrm{GaP}$ QD with a WL are illustrated in the linear case $\left(\mathrm{P}_{1}\right)$ a), the quadratic case $\left.\left(\mathrm{P}_{2}\right) \mathrm{b}\right)$, the sum of both contributions $P_{1}$ plus $\left.P_{2} c\right)$, and for the linear piezoelectric experimental constants $P_{e x} d$ ). 
FIG. 1.

a)
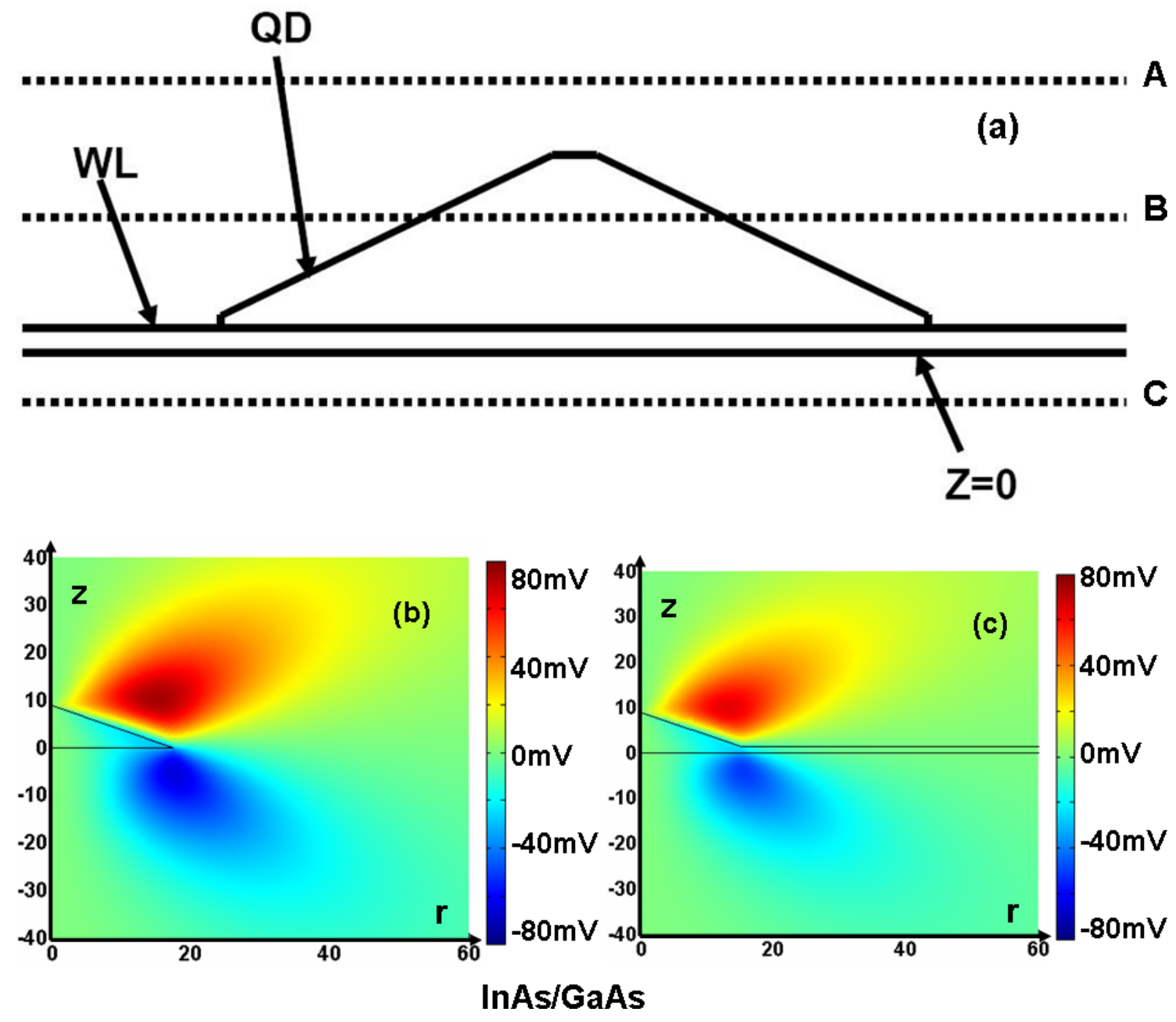
FIG. 2

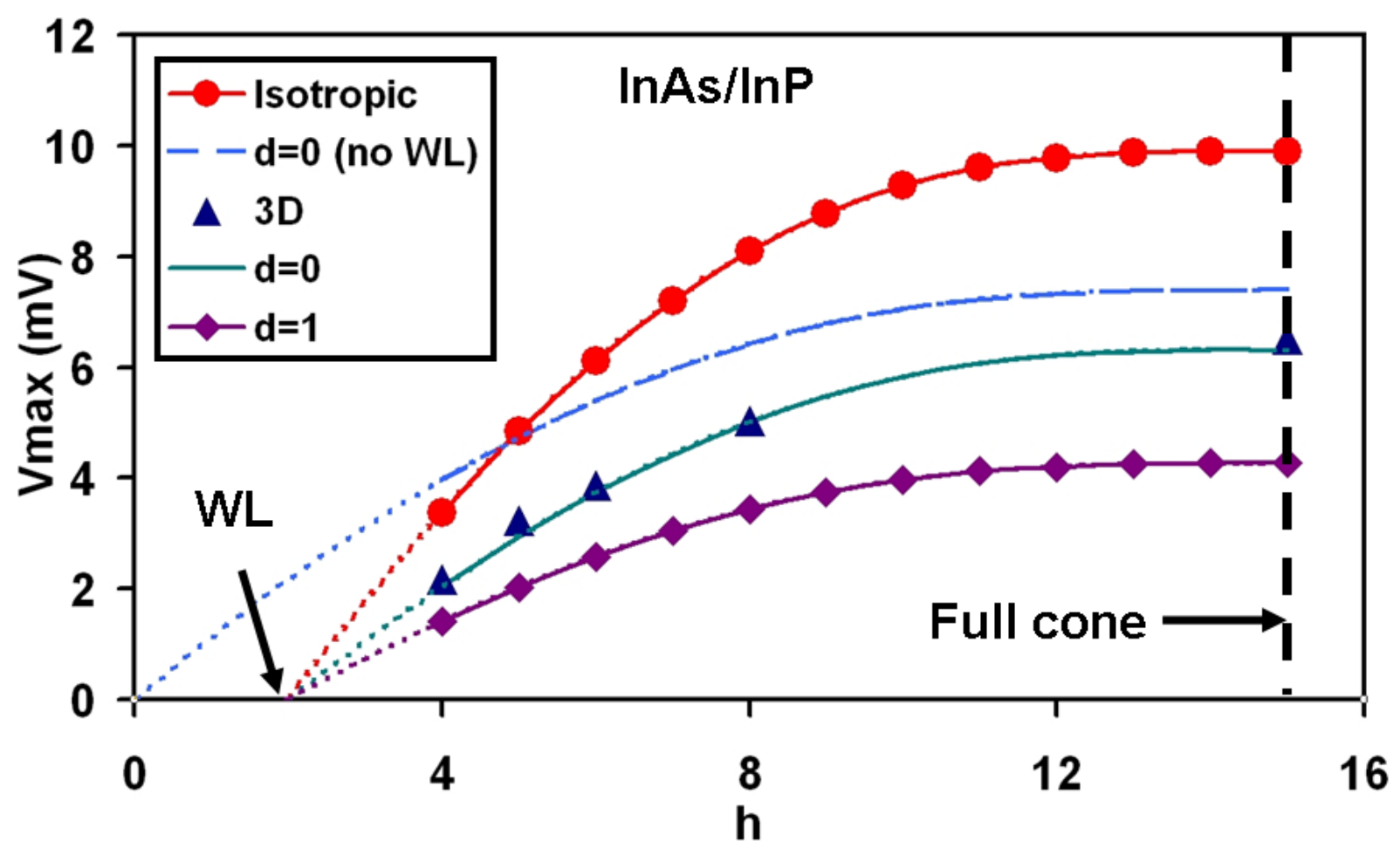


FIG.3

(a)

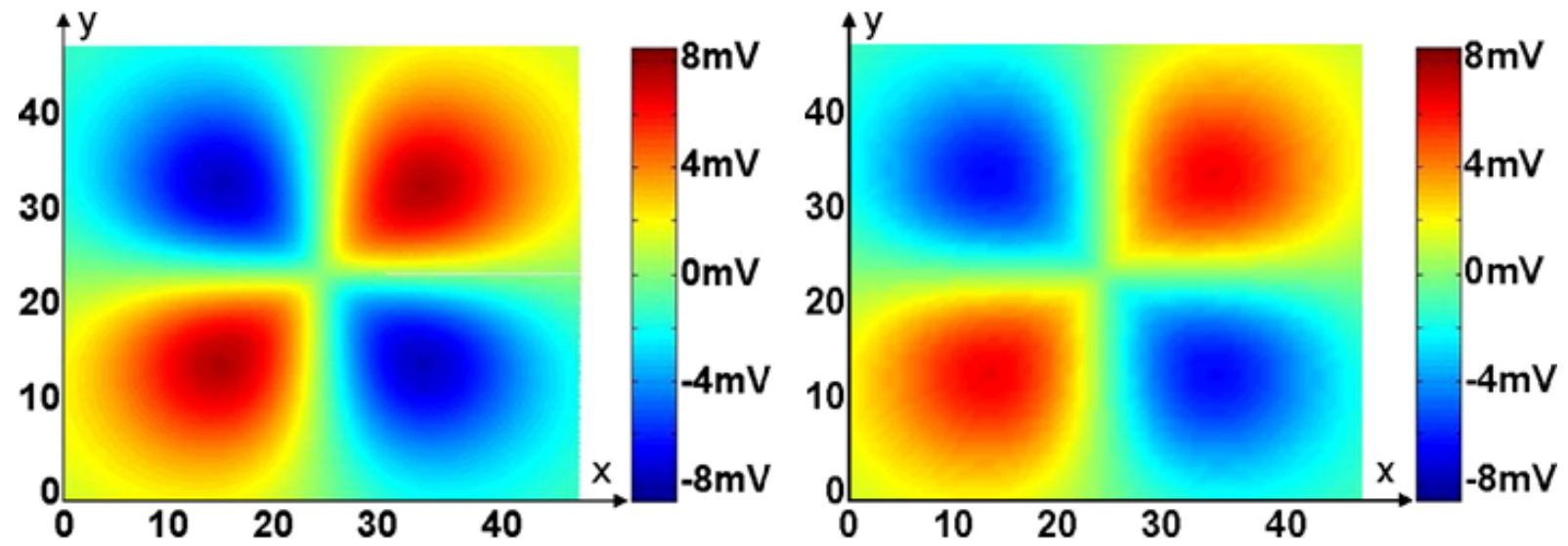

(b)

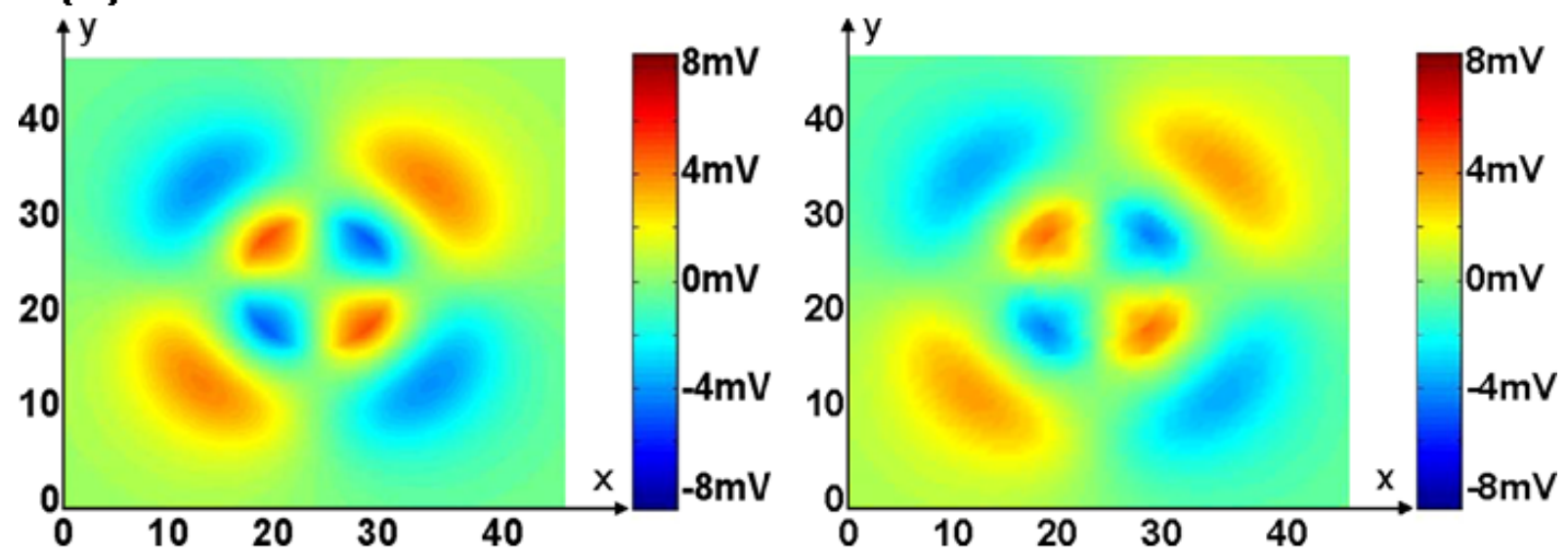

(c)

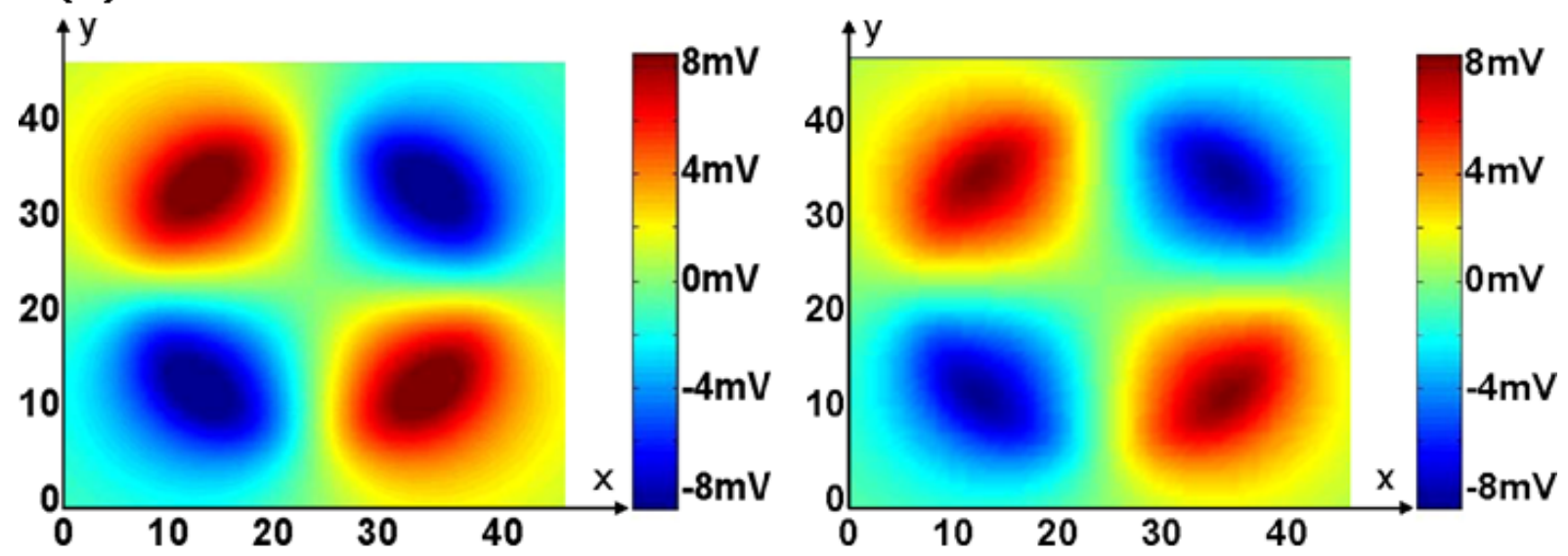

InAs/InP 
FIG. 4.
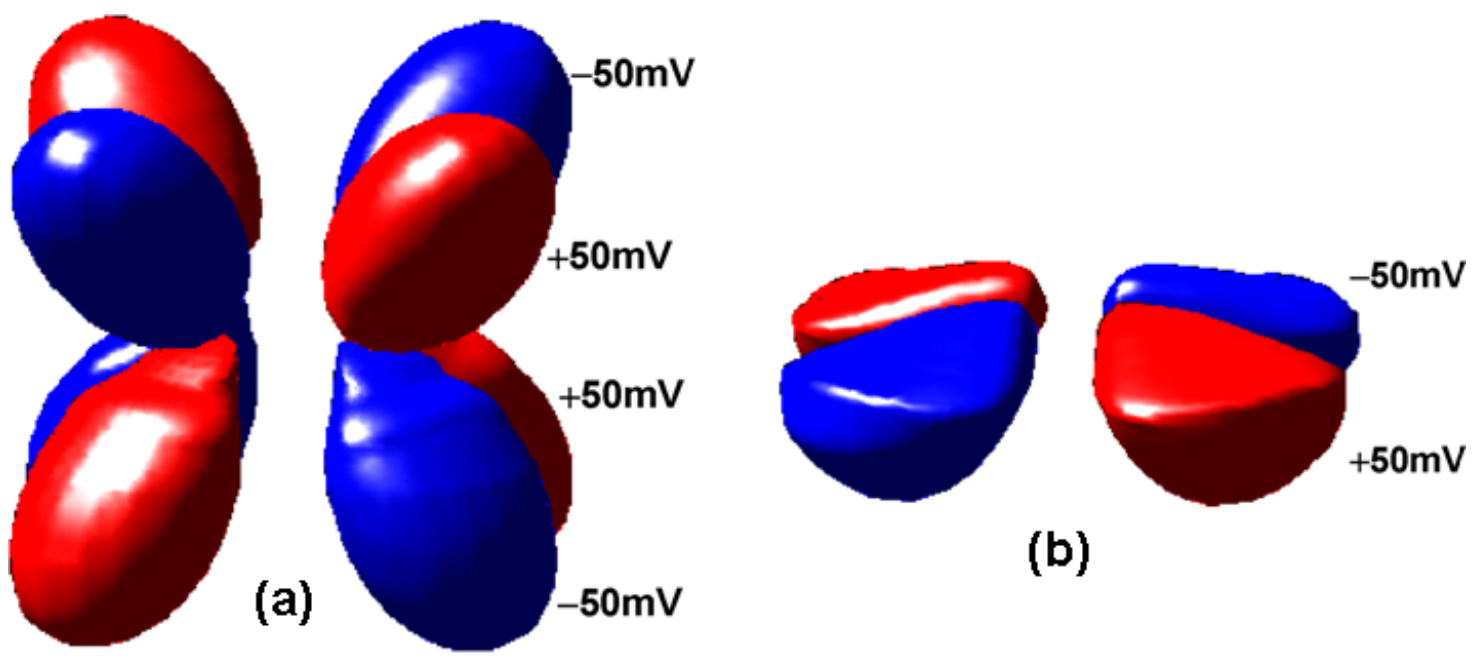

(b)
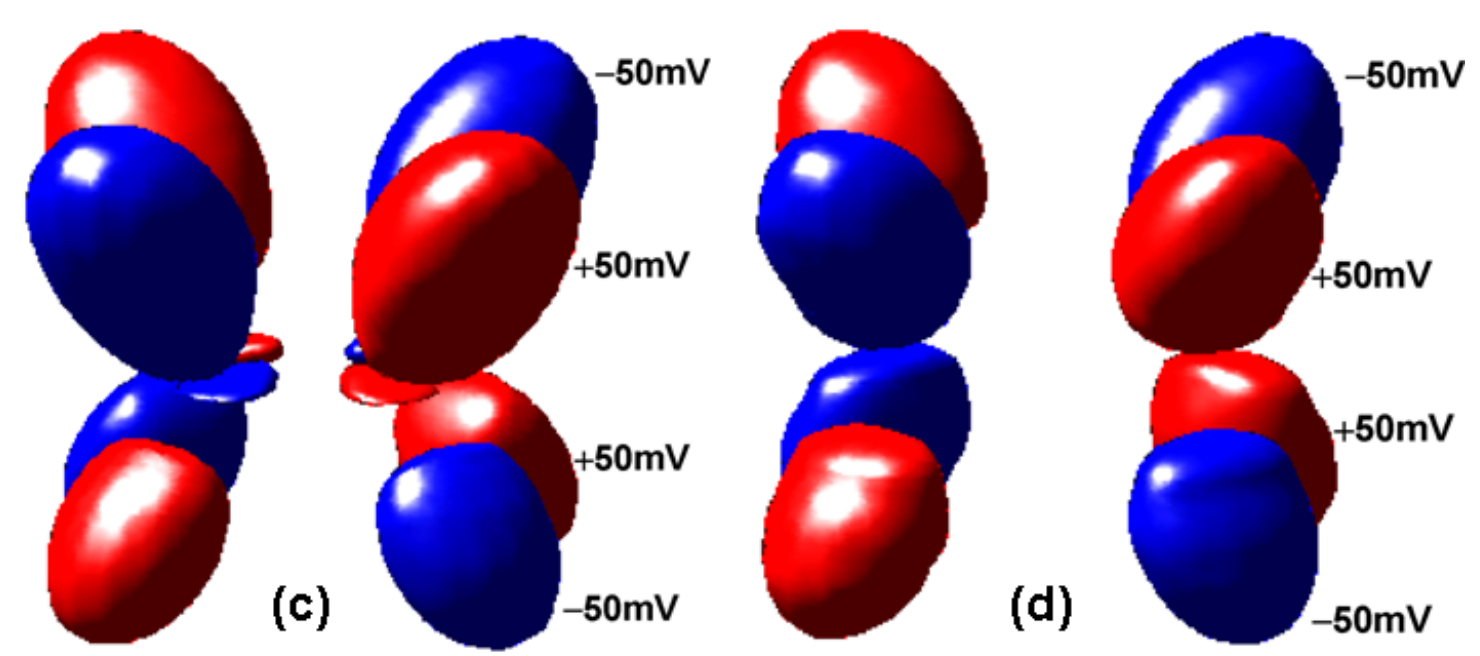

InAs/GaP

(d) 